\title{
Photoactivable Polymers Embedded with Cadmium-free \\ Quantum Dots and Crystal Violet: Efficient Bactericidal Activity against Clinical Strains of Antibiotic-Resistant Bacteria
}

Ethel Owusu, Alexander J. MacRobert, Elaine Allan, Ivan P. Parkin, Elnaz Yaghini, and Imad Naasani

ACS Appl. Mater. Interfaces, Just Accepted Manuscript • DOI: 10.1021/acsami.9b02109 • Publication Date (Web): 11 Mar 2019

Downloaded from http://pubs.acs.org on March 18, 2019

\section{Just Accepted}

"Just Accepted" manuscripts have been peer-reviewed and accepted for publication. They are posted online prior to technical editing, formatting for publication and author proofing. The American Chemical Society provides "Just Accepted" as a service to the research community to expedite the dissemination of scientific material as soon as possible after acceptance. "Just Accepted" manuscripts appear in full in PDF format accompanied by an HTML abstract. "Just Accepted" manuscripts have been fully peer reviewed, but should not be considered the official version of record. They are citable by the Digital Object Identifier (DOI®). "Just Accepted" is an optional service offered to authors. Therefore, the "Just Accepted" Web site may not include all articles that will be published in the journal. After a manuscript is technically edited and formatted, it will be removed from the "Just Accepted" Web site and published as an ASAP article. Note that technical editing may introduce minor changes to the manuscript text and/or graphics which could affect content, and all legal disclaimers and ethical guidelines that apply to the journal pertain. ACS cannot be held responsible for errors or consequences arising from the use of information contained in these "Just Accepted" manuscripts. 


\title{
Photoactivable Polymers Embedded with Cadmium-free Quantum Dots and Crystal Violet: Efficient Bactericidal Activity against Clinical Strains of Antibiotic-Resistant Bacteria
}

\author{
Ethel G. A. Owusu, ${ }^{\dagger}$,,$\S$ Alexander J. MacRobert, ${ }^{\star *}$ Imad Naasani, ${ }^{\#}$ Ivan P. Parkin, ${ }^{\ddagger}$ \\ Elaine Allan, $\S$ Elnaz Yaghini ${ }^{*}$
}

† UCL Division of Surgery and Interventional Science, University College London, Charles Bell House, 43-45 Foley Street, London W1W 7TS, United Kingdom

¥ Materials Chemistry Research Centre, Department of Chemistry, University College London, 20 Gordon Street, London WC1H 0AJ, United Kingdom

$\S$ Department of Microbial Diseases, UCL Eastman Dental Institute, University College London, 256 Gray's Inn Road, London WC1X 8LD, United Kingdom

\# Nanoco Technologies Ltd, 46 Grafton Street, Manchester M13 9NT, United Kingdom

*E-mail: a.macrobert@ucl.ac.uk; elnaz.yaghini@ucl.ac.uk

\begin{abstract}
The rising incidence of antibiotic-resistant infections from contaminated surfaces in hospitals or implanted medical devices has led to increasing interest in new antibacterial surfaces. Photoactivatable surfaces that can generate cytotoxic reactive oxygen species under exposure to ambient light is a promising approach to inactivation of surface-borne microorganisms. There is growing interest in the use of quantum dots (QDs) as lightharvesting agents for photobactericidal applications but the cadmium in commonly used QDs will restrict clinical application. Herein, the photobactericidal activity of novel polyurethane substrates containing cadmium-free quantum dots was tested against clinical multi-drug resistant Gram-positive and Gram-negative bacterial strains: methicillin-resistant Staphylococcus aureus (MRSA) and a carbapenemase-producing strain of Escherichia coli $(\mathrm{E}$. coli). To enhance the capacity for reactive oxygen species generation, QDs were incorporated into the polymer with a photosensitising dye, crystal violet. Close proximity between the QD and dye enables electron and energy transfer processes leading to generation of cytotoxic singlet oxygen and superoxide radicals. A QD solution in cyclohexane was premixed with a solution of $\mathrm{CV}$ in the more polar solvent, dichloromethane, to promote the formation of QD-CV nanocomposite complexes via CV adsorption. This solution was then used to embed the quantum dots and crystal violet into medical grade polyurethane via swellencapsulation. The combination of QD and CV elicited significant synergistic antibacterial activity under visible light against MRSA within $1 \mathrm{~h}(99.98 \%$ reduction) and $\mathrm{E}$. coli within $4 \mathrm{~h}$ (99.96\% reduction). Photoluminescence lifetime and singlet oxygen phosphorescence measurements demonstrated interaction between the quantum dots and the crystal violet occurs within the polymer that can lead to enhanced generation of reactive oxygen species. Strong inhibition of kill was observed using the superoxide scavenger, superoxide dismutase. The efficacy of these QD-CV polymer substrates that can harvest light across the visible spectrum, against multi-drug resistant bacteria demonstrates the feasibility of this approach.
\end{abstract}

Key words: antibacterial, surfaces, polymer, light-activated, photodynamic therapy, quantum dots, crystal violet, reactive oxygen species 


\section{Introduction}

Each year, over 4 million patients in the European Union and 2 million in the United States acquire a healthcare-associated infection (HAl), contributing to roughly 110,000 and 99,000 associated deaths respectively. ${ }^{1-2}$ Healthcare-associated infections which are also referred to as nosocomial infections lead to prolonged suffering for patients and in many cases longer hospital stays. The rising incidence of antimicrobial resistance (AMR) whereby bacteria become resistant to previously effective medications further exacerbates the impact of HAls: more than $70 \%$ of the bacteria that causes HAls are resistant to at least one commonly prescribed antibiotic. ${ }^{3}$ Excessive and often unnecessary antibiotic use increases the selective pressure on bacterial populations, creating a pool of resistant genes some of which can transfer horizontally between populations and create multi-drug resistant (MDR) HAls that are increasingly difficult to treat, lead to significant patient morbidity and substantial costs for healthcare systems. ${ }^{4}$

Among the most common sources of infectious agents causing HAls are inanimate surfaces and objects (for example, patient room touch surfaces, medical equipment and devices) that have become contaminated. ${ }^{5}$ One strategy to reduce the nosocomial reservoir of bacteria is the use of self-disinfecting antimicrobial surfaces. The use of photosensitisers (PS) of low toxicity such as toluidine blue $\mathrm{O}$ (TBO), methylene blue (MB) and crystal violet (CV) in photodynamic therapy (PDT) has been shown to be effective against a wide range of microbes. ${ }^{6-9}$ Endowment of polymers, which are widely used in medical devices, with antibacterial activity has been achieved by encapsulation with photosensitisers and irradiation with visible non-ionising light. ${ }^{10-14}$ Bactericidal activity is mediated by reactive oxygen species (ROS) generated via photodynamic processes: illumination with light at the appropriate wavelength activates the photosensitiser to an excited singlet state which subsequently converts to a long-lived triplet state which leads to generation of ROS via photoelectron transfer (PET) (Type I) or energy transfer (Type II) pathways. In Type I, photo-induced redox result in the generation of free radicals such as superoxide and hydroxyl radicals. The Type II pathways involve direct energy transfer from the triplet state to molecular oxygen generating highly reactive singlet oxygen $\left({ }^{1} \mathrm{O}_{2}\right) \cdot{ }^{15} \mathrm{ROS}$ such as superoxide, hydroxyl radicals and singlet oxygen can oxidise biological substrates thereby inducing irreversible cellular membrane damage and enzyme deactivation, ultimately causing cell death. Nevertheless, despite their efficacy, the utility of PS as antimicrobial agents can be limited by their narrow absorption bands and susceptibility to photobleaching. Quantum dots (QDs) are emerging as a new class of versatile photoluminescent probes for many biomedical applications such as drug delivery, sensors, cancer therapy and imaging. ${ }^{16-19}$ Compared to organic dyes including conventional photosensitisers, QDs have intense and broad light absorption, high photobleaching threshold and photoluminescence (PL) quantum yield and long photoluminescence lifetimes. ${ }^{20}$ Additionally, by tailoring QDs shape, size and composition, QDs can be engineered to emit at wavelengths that can span the entire spectrum. ${ }^{21}$

These unique photophysical properties and their broad-band light harvesting capability have prompted interest in the use of quantum dots as photosensitising agents. ${ }^{22}$ Under high power visible light doses, QDs have been effective in inhibiting the growth of a range of Gram-positive and Gram-negative bacteria through the generation of ROS. ${ }^{23-26}$ As with conventional photosensitising organic dyes, QDs may generate ROS via Type I and Type II pathways and there are several reports of direct formation of superoxide radical anions by photoelectron transfer (PET) from photoactivated cadmium and indium-based QDs. ${ }^{23-24,} 27$ Dismutation of superoxide can generate hydrogen peroxide which in turn can generate highly reactive hydroxyl radicals via the iron-catalysed Fenton process. With the exception of some graphene dots reported in the literature,,$^{28-29}$ QDs exhibit very low quantum yields of ${ }^{1} \mathrm{O}_{2}$ (regarded as the most potent ROS), compared to clinically used photosensitisers. ${ }^{27,}{ }^{30}$ Conjugation of QDs with photosensitising dyes has been shown to significantly increase singlet oxygen efficiencies. In this configuration, excited QDs can act as energy donors and excite PS acceptors to their 
excited singlet state by transferring energy via the mechanism known as Förster Resonance Energy Transfer (FRET). ${ }^{22,31-32}$

A major drawback with their biomedical use is that QDs generally contain restricted heavy metals such as cadmium which severely hinders the clinical translation of such QDs owing to the possible release of toxic $\mathrm{Cd}^{2+}$ ions which can also induce oxidative stress via generation of ROS. ${ }^{33-34}$ Recently, we and others have reported on the use of indium-based cadmium-free QDs and demonstrated their safety in rodent models, which should facilitate their translation to clinical use..$^{35-36}$ Herein, we report the development of novel photoactivatable antimicrobial surfaces based on polyurethane embedded with a combination of crystal violet (CV) and new cadmium-free indium-based QDs (CFQD ${ }^{\circledR}$ nanoparticles). Polyurethane was selected since it is widely used for medical device applications. We hypothesised that the addition of QDs to $\mathrm{CV}$ encapsulated in polymer would boost antimicrobial activity by increasing production of cytotoxic reactive oxygen species. The QD-CV complexes were produced by adapting the simple non-covalent incorporation technique, namely 'swell-encapsulation-shrink'. ${ }^{13}$ The antibacterial activity of QD-CV embedded polyurethane substrates was tested against representative multidrug resistant nosocomial Gram-positive and Gram-negative pathogens.

\section{Methods and Materials \\ Materials}

$\mathrm{CFQD}^{\circledR}$ nanoparticles dispersed in cyclohexane were synthesised and provided by Nanoco Technologies Ltd (Manchester, UK). Medical grade polyurethane sheets (thickness $0.8 \mathrm{~mm}$ ) were purchased from American Polyfilm Inc. (Branford, USA). Crystal violet dye and solvents were purchased from Sigma-Aldrich (UK).

\section{Synthesis of CFQD ${ }^{\circledR}$ nanoparticles}

Indium-based QDs (CFQD ${ }^{\circledR}$ nanoparticles) dispersed in cyclohexane with peak photoluminescence at $620 \mathrm{~nm}$ were synthesised and provided by Nanoco Technologies Ltd. using proprietary synthesis procedures based on the molecular seeding process. ${ }^{37}$ Briefly, a $\mathrm{ZnS}$ molecular cluster $\left[\mathrm{Et}_{3} \mathrm{NH}_{4}\right]\left[\mathrm{Zn}_{10} \mathrm{~S}_{4}(\mathrm{SPh})_{16}\right]$ was heated in the presence of indium myristate $\left(\operatorname{In}(\mathrm{MA})_{3}\right)$ and Tris(trimethylsilyl)phosphine $\left((\mathrm{TMS})_{3} \mathrm{P}\right)$ in a medium of di-n-butylsebacate ester. The indium-based alloyed cores were washed with hydrofluoric acid and then heated in a solution of zinc acetate and Bis(trimethylsilylmethyl) sulfide $\left((\mathrm{TMS})_{2} \mathrm{~S}\right)$ in butylsebacate ester to form a coating layer of $\mathrm{ZnS} .{ }^{35}$ Finally, the nanoparticles were isolated by precipitation in acetone and then re-dispersed in cyclohexane. The resulting nanoparticles had surface capping ligands of mainly myristate and butyl sebacate, which will confer an electronegative polarity, and were isolated by precipitation in acetone and then re-dispersed in cyclohexane. The quantum efficiencies of the final unfunctionalised indium-based nanoparticle material ranged from $73 \%$ to $79 \%$ in cyclohexane. The molecular weight of the CFQD ${ }^{\circledR}$ nanoparticles is $\sim 500 \mathrm{kDa}$, therefore a $1 \mathrm{mg} / \mathrm{mL}$ concentration is approximately equivalent to $2 \mu \mathrm{M}$.

\section{Incorporation of nanoparticles and dye into polymer}

The CFQD ${ }^{\circledR}$ nanoparticles and the CV were incorporated non-covalently into polyurethane via a simple dipping process known as 'swell-encapsulation-shrink'. ${ }^{13}$ Stock QDs solutions were prepared in cyclohexane (Cy) with a concentration of $2 \mathrm{mg} / \mathrm{mL}$ and CV stock solutions were prepared in dicholoromethane (DCM) with a concentration of $1 \mathrm{mM}$. Dipping solutions were prepared to have final concentrations of either $1 \mathrm{mg} / \mathrm{mL} \mathrm{QD}+0.5 \mathrm{mM} \mathrm{CV} ; 1 \mathrm{mg} / \mathrm{mL} \mathrm{QD}$; or $0.5 \mathrm{mM} \mathrm{CV}$ in a solvent system of $1: 1$ cyclohexane: dichloromethane, to produce polymer samples with QDs and CV (QD + CV PU), QDs only (QD PU) and CV only (CV PU). In all cases, a $1 \mathrm{~cm}^{2}$ polymer squares were placed into the appropriate solutions and left to swell in the dark for $24 \mathrm{~h}$ inside a closed bottle containing $10 \mathrm{~mL}$ of dipping solution. Subsequently, samples were left to dry in the dark at room temperature for $24 \mathrm{~h}$, washed with $\mathrm{dH}_{2} \mathrm{O}$ and air dried. As controls, polymer samples were swollen in pure 1:1 Cy:DCM solvent. 


\section{Material Characterisation}

Transmission electron microscopy (TEM) of quantum dots

A solution of the quantum dots in cyclohexane was drop cast onto 300 mesh carbon coated copper grid (Agar Scientific) and air-dried prior to imaging. High resolution transmission electron microscope (TEM) images were acquired using a JEOL 2100 TEM with a LaB6 source operating at an acceleration voltage of $200 \mathrm{kV}$. Micrographs were taken on a Gatan Orius charge-coupled device (CCD) camera with Digital Micrograph software. Particle size analysis was carried out Gatan Suite software.

\section{Spectroscopic measurements}

UV-vis absorption spectra of suspensions and polymer substrates were recorded using dual beam spectrophotomers (PerkinElmer Lambda 950 or Shimadzu UV-2600). Emission spectra of suspensions were recorded with a spectrofluorimeter (Horiba FMax4 Fluorimeter). Quartz cuvettes were employed of $1 \mathrm{~cm}$ path length. For the emission spectroscopy measurements of the polyurethane substrates, which are strongly light-scattering, samples were mounted diagonally at $45^{\circ}$ incidence to the excitation beam, and a long-pass Schott filter (RG435) was installed in the emission port to filter out the blue excitation scattered light. The overlap integral $J(\lambda)$ for the FRET study was calculated using open source software (FluorTools.com).

\section{Singlet Oxygen Phosphorescence Measurements}

The singlet oxygen phosphorescence at $1270 \mathrm{~nm}$ of modified medical grade polyurethane incorporated with QD and/or CV was detected using time-resolved photon counting. For detection in the near-IR, a thermoelectrically cooled photomultiplier (model H10330-45, Hamamatsu Photonics Ltd, Hertfordshire, UK) was used, and the emission was collected via a series of lenses from the cuvette in combination with a long-pass (950 nm cut-on, Andover Corp., USA) and a band-pass filter centred at $1270 \mathrm{~nm}$ (Interferenzoptik Electronik $\mathrm{GmbH}$, Germany). Polyurethane samples were mounted diagonally in a quartz cuvette and irradiated using a $532 \mathrm{~nm} \mathrm{Nd}$ :YAG laser (Lumanova $\mathrm{GmbH}$, Germany) with the beam axis aligned at $45^{\circ}$ to the surface plane of the sample in order to optimise detection of ${ }^{1} \mathrm{O}_{2}$ within the polymer. The laser was pulsed at a repetition rate of $3 \mathrm{kHz}$ and a pulse length of $3 \mathrm{~ns}$ and a fast photodiode ( $1 \mathrm{~ns}$ rise time, Becker-Hickl, Germany) was used to synchronise the laser pulse with the photon counting detection system. Neutral density filters was used to attenuate the laser power to $2 \mathrm{~mW}$. The photon counting equipment consisted of a PC-mounted multiscaler board (model MSA-300, Becker-Hickl, Germany) and a pre-amplifier (Becker-Hickl, Germany) which gave a resolution of $5 \mathrm{~ns}$ per channel. Time-resolved phosphorescence measurements were accumulated by the multiscaler board at a $0.1 \mu \mathrm{s}$ bin width and the signals were analysed using FluoFit software (PicoQuant $\mathrm{GmbH}$, Germany) to extract the lifetime parameters.

\section{Time-Resolved Lifetime Measurements}

Photoluminescence lifetime of QDs was measured using time-correlated single photon counting (TCSPC). Solutions of QDs and QD-CV complexes at various CV concentrations (fixed QD concentration) were prepared and placed in a $1 \mathrm{~cm}$ optical path quartz cuvette. A pulsed laser diode module Edinburgh instrument Ltd., UK model EPL-405 was used to excite the samples at $405 \mathrm{~nm}$ at a $1 \mathrm{MHz}$ repetition rate (EPL- 405, Edinburgh Instruments Ltd., UK). The emission was detected using a fast multialkali photomultiplier module (model H5773-04, Hamamatusu Photonics K.K., Japan) via a long-pass filter (OG510, Schott, UK) and a monochromator (model M300, Bentham Instrument Ltd, UK). A Lyot depolarizer (Thorlabs Ltd, Ely, UK) was incorporated to minimise any polarisation anisotropy artefacts. TCSPC was carried out using a PC-mounted TCSPC board (TimeHarp 260, PicoQuant GmbH, Germany) and lifetimes were derived using Fluofit software (PicoQuant $\mathrm{GmbH}$, Germany). The Instrument Response Function (IRF) was obtained from a non-fluorescent scattering Ludox solution (Sigma-Aldrich, UK). Optimum fitting with minimisation of the residuals was confirmed using a Chi-squared value $\chi^{2}<1.4$. The photoluminescence lifetime of QDs was also measured after embedding of QDs and/or CV in the polymer. The substrates were mounted 
on microscope glass slides and signals were detected as described for QD and QD-CV solutions above.

\section{Bacterial Strains}

Methicillin-resistant Staphylococcus aureus (MRSA NCTC 13143) was obtained from the National Collection of Type Cultures (PHE, Colindale); a clinical strain of epidemic methicillinresistant Staphylococcus aureus (EMRSA 4742) was obtained from P. Wilson, University College London Hospital; and the multi-drug resistant (MDR) carbapenemase-producing clinical strain of Escherichia coli (E. coli 1030) was obtained from J. Wade, King's College Hospital, London). These organisms were stored at $-70^{\circ} \mathrm{C}$ in Brain-Heart Infusion broth (BHI, Oxoid) containing $20 \%(\mathrm{v} / \mathrm{v})$ glycerol and propagated onto either MacConkey agar (MAC, Oxoid) in the case of $E$. coli or mannitol salt agar (MSA, Oxoid) in the case of MRSA, for a maximum of 2 subcultures at intervals of 2 weeks.

\section{Antibacterial Activity}

The following $1 \mathrm{~cm}^{2}$ polyurethane (PU) squares were used in the microbiology experiments:

i. solvent treated polyurethane (control PU)

ii. $\quad$ crystal violet encapsulated polyurethane (CV PU)

iii. Quantum dot encapsulated polyurethane (QD PU)

iv. Quantum dot and crystal violet encapsulated polyurethane (QD + CV PU)

For testing of each strain, Brain-Heart Infusion broth (BHI, Oxoid) was inoculated with 1 bacterial colony and cultured in air at $37^{\circ} \mathrm{C}$ for $18 \mathrm{~h}$ with shaking, at $200 \mathrm{rpm}$. The bacterial pellet was recovered by centrifugation, $\left(20^{\circ} \mathrm{C}, 2867.2 \mathrm{~g}, 5 \mathrm{~min}\right)$, washed in phosphate buffered saline (PBS) $(10 \mathrm{~mL})$, and centrifuged again to recover the pellet $\left(20^{\circ} \mathrm{C}, 2867.2 \mathrm{~g}, 5 \mathrm{~min}\right)$, and the bacteria were finally re-suspended in PBS $(10 \mathrm{~mL})$. The washed suspension was diluted 1000 -fold to obtain an inoculum of $\sim 10^{6} \mathrm{cfu} / \mathrm{mL}$. In each experiment, the inoculum was confirmed by plating 10 -fold serial dilutions on agar for viable counts. Triplicates of each polymer sample type were inoculated with the inoculum $(25 \mu \mathrm{L})$ and covered with a sterile coverslip $\left(2.2 \mathrm{~cm}^{2}\right)$. The samples were then incubated in the light and dark for up to $4 \mathrm{~h}$. Under light conditions, a white fluorescent light source (General Electric, USA) ${ }^{25}$, emitting at an average light intensity of $6000 \pm 990$ lux measured at $25 \mathrm{~cm}$ from the samples, was employed. After incubation, the inoculated samples and coverslips were added to PBS (450 $\mu \mathrm{L})$ and mixed using a vortex mixer. The neat suspension and 10 -fold serial dilutions were plated on agar for viable counts and incubated aerobically at $37^{\circ} \mathrm{C}$ for $24 \mathrm{~h}$ (E. coli) or $48 \mathrm{~h}$ (MRSA).

\section{Detection of Superoxide}

To investigate the mechanism of bactericidal activity against bacteria, superoxide dismutase (SOD) in PBS (50 U/mL) was added to the bacterial suspension and exposed to the polyurethane substrates using the protocol described above to deactivate any superoxide radicals emanating from the polymer surface. SOD from bovine erythrocytes was purchased from Sigma-Aldrich, UK and filter sterilised using a 0.2 $\mu \mathrm{m}$ PES syringe filter (VWR, UK).

\section{Statistical Analysis}

Each experiment contained a minimum of two technical replicates and the experiments were carried out three times. The statistical significance, in both light and dark conditions, was analysed using independent sample t-test of the following comparisons:
a. Control vs. inoculum
b. Crystal violet alone vs. control
c. Quantum dot alone vs. control
d. Quantum dot with crystal violet combination vs. crystal violet alone
e. Quantum dot with crystal violet combination vs. control 
Results were considered significantly different for $\mathrm{P}<0.05$. Error bars show the standard deviation from the mean.

\section{Results \& Discussion}

\section{Characterisation}

TEM images of the CFQD ${ }^{\circledR}$ nanoparticles in solution showed the particles to be spherical and mono-disperse (Fig. 1A). Analysis of over 150 particles gave an average size of $3.6 \mathrm{~nm}$ with a narrow size distribution SD of $\pm 0.6 \mathrm{~nm}$ (Fig. 1B).
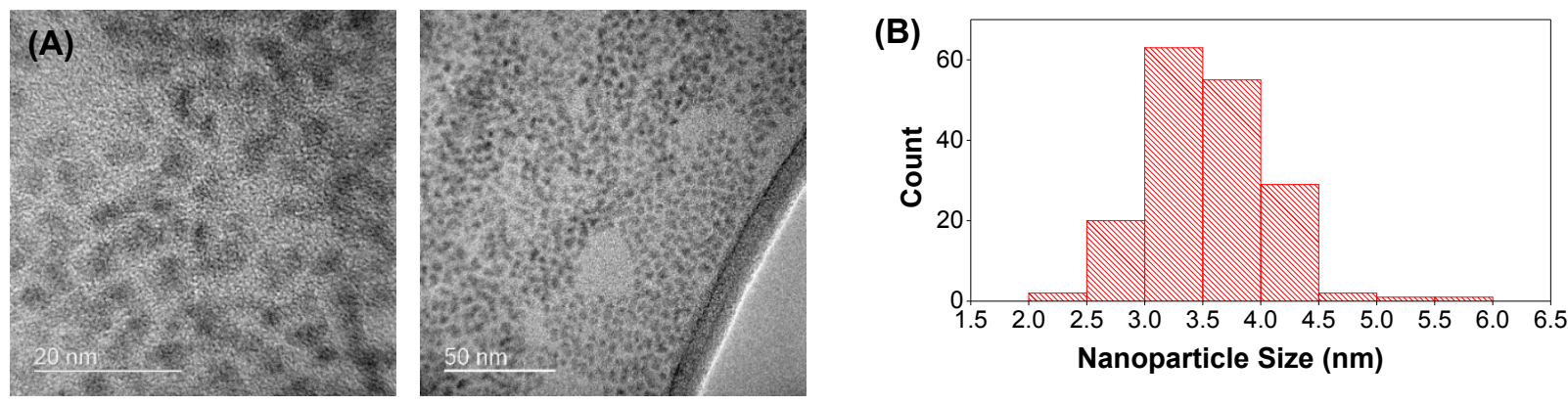

Figure 1. (A) High resolution TEM image of $C F Q D^{\circledR}$ nanoparticles. (B) Size distribution of $C F Q D^{\circledR}$ nanoparticles from TEM

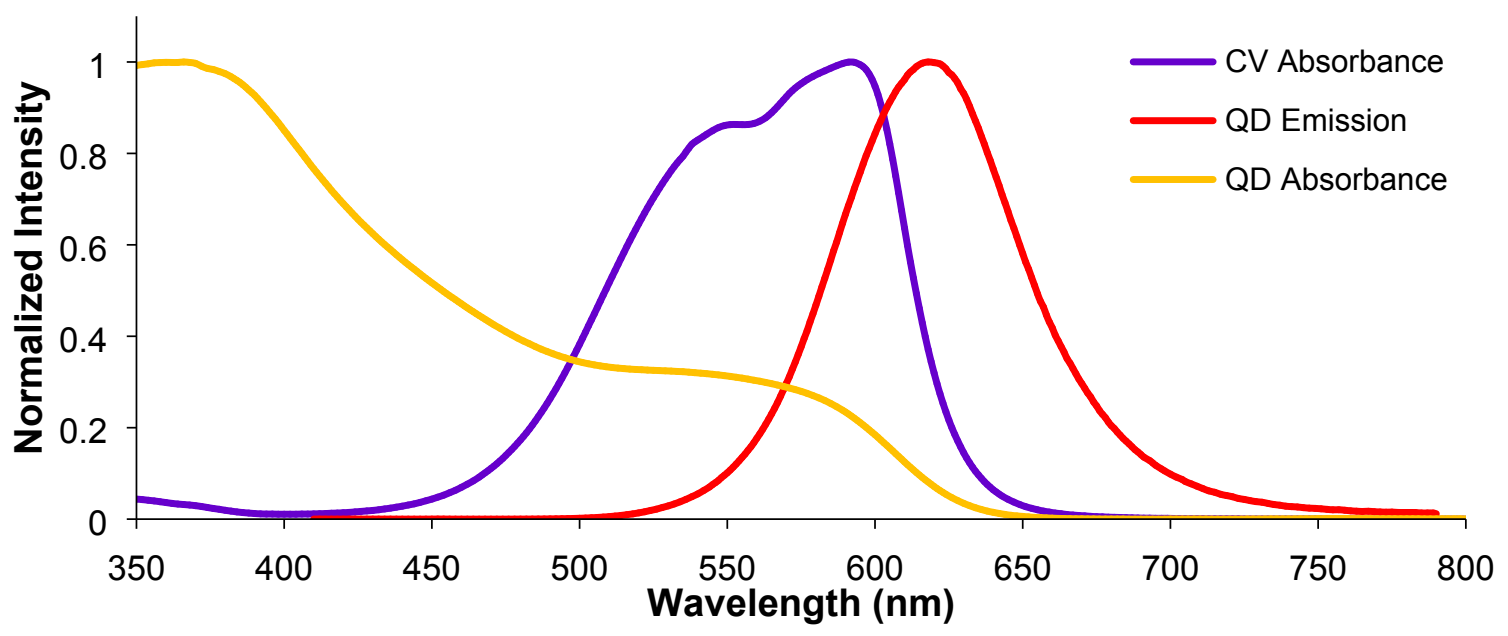

Figure 2. Normalized absorbance (yellow line) and emission spectra (red line) of CFQD ${ }^{\circledR}$ nanoparticles and absorbance spectrum of crystal violet (purple line) in 1:1 cyclohexane/dichloromethane solvent. The emission spectrum of $\mathrm{CV}$ is not shown as it exhibits negligible fluorescence in low viscosity solutions.

The QD emission spectrum is characterised by a symmetric profile with a full-width at half maximum of about $60 \mathrm{~nm}$ and a sharply defined maximum emission of $620 \mathrm{~nm}$. The absorption spectrum of the QD showed broad peaks in the visible region up to about $600 \mathrm{~nm}$ and strong absorption in the blue region (Fig. 2). CV is a highly coloured triphenylamine dye with an extensive history as an anti-bacterial and anti-fungal agent, which we have used in previous antimicrobial polymer studies in combination with gold and zinc oxide nanoparticles. ${ }^{14,} 38 \mathrm{CV}$ is also inexpensive, chemically stable ${ }^{39}$ and has been widely used as a therapeutic agent in the clinic for conditions such as oral candiadis (thrush), eczema and as a blood additive to prevent transmission of Chagas' disease. ${ }^{10,39-41}$ The absorption spectrum of crystal violet is narrow compare to the QD spectrum and has an overlapped doublet spectrum with the 
maximum absorption peak at $590 \mathrm{~nm}$ and a shoulder around $550 \mathrm{~nm}$ (Fig. 2). ${ }^{42} \mathrm{CV}$ dimerisation in solution leads to small blue shifts in the spectrum. ${ }^{43}$ The emission spectrum of $\mathrm{CV}$ was not recorded in solution since most arylmethane dyes have typically low emission due to the strong dependence of fluorescence quantum yields on solvent viscosity. In low viscosity fluid solvents, the arylmethane groups can rotate freely resulting in the formation of twisted intramolecular charge transfer (TICT) states with negligible fluorescence quantum yields due to rapid radiationless decay to the ground state. However, this molecular rotor effect is diminished when $\mathrm{CV}$ is dissolved in high viscosity solvents or bound to proteins and CV fluorescence from the first excited singlet state can then be measured ${ }^{44-46}$ Likewise, when CV is encapsulated in polymer where the spatial constraint imposed by the matrix will restrict the rotor effect, the CV fluorescence is evident. ${ }^{14,46}$

\section{Incorporation of Nanoparticle and Dye into Polymer}

The swell-encapsulation-shrink technique ${ }^{12-13}$ that we have previously employed as a two-step process was modified to a one-step process in order to improve co-localisation of the QD and $\mathrm{CV}$ in the polymer and complex formation. This was achieved by mixing the QDs and CV in a miscible 1:1 cyclohexane/dichloromethane solvent system as illustrated in Figure 3A. A mixed solvent system was required since the QDs are soluble in cyclohexane whereas CV was not directly soluble. The CV was therefore first dissolved in DCM and subsequently this solution was mixed with the cyclohexane QD solution. The polymer samples were then treated with this solution for $24 \mathrm{~h}$ resulting in purple-stained samples which after drying were used for antimicrobial testing. QD photoluminescence and CV fluorescence measurements of the polymers after modification confirmed uptake of the QD and/or CV (Fig. 3B, 3C).
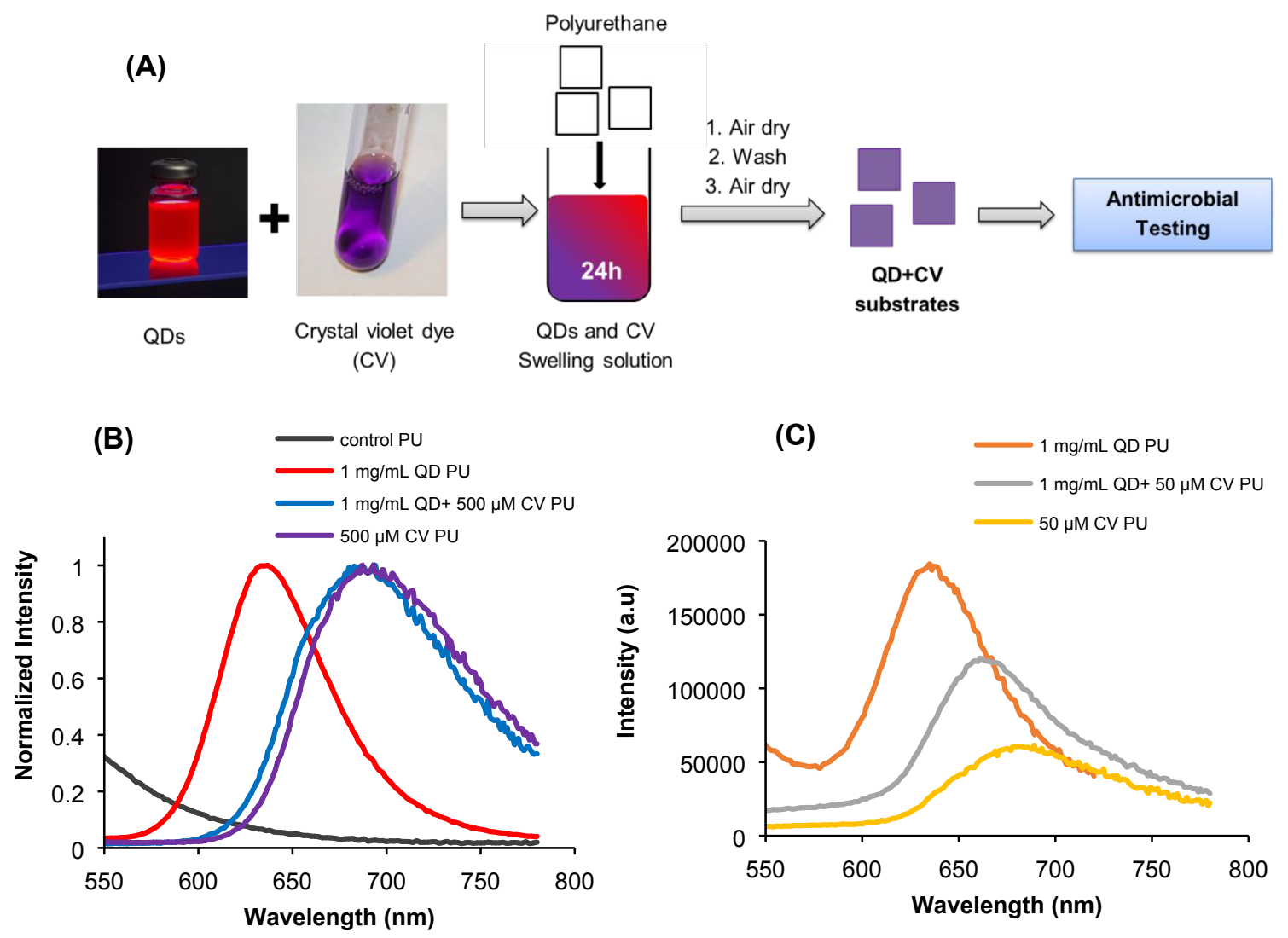

Figure 3. (A) Scheme shows the preparation of QD nanoparticle and crystal violet encapsulated polyurethane samples. Squares indicate the polyurethane squares used. (B) Normalized emission spectra of polyurethane substrates confirming uptake of QD and/or CV. Emission of the control polyurethane (grey line), polyurethane encapsulated with $1 \mathrm{mg} / \mathrm{mL}$ QDs (red line), $500 \mu \mathrm{M}$ CV (purple line) and a combination of $1 \mathrm{mg} / \mathrm{mL}$ QDs and $500 \mu \mathrm{M} \mathrm{CV}$ (blue line) measured at excitation wavelength of $400 \mathrm{~nm}$. (C) Emission spectra of polyurethane samples doped with a lower CV concentration 
following excitation at $400 \mathrm{~nm}: 1 \mathrm{mg} / \mathrm{mL}$ QDs (orange line), $50 \mu \mathrm{M} \mathrm{CV}$ (yellow line) and a combination of $1 \mathrm{mg} / \mathrm{mL}$ QDs and $50 \mu \mathrm{M} \mathrm{CV}$ (grey line).

In the CV-doped polymer, the CV emission spectrum appears to be markedly red-shifted to $\sim 700 \mathrm{~nm}$ at higher concentrations (Fig. 3B) compared to that observed for polymer samples doped at lower concentrations as shown in Figure S1: for $5 \mu \mathrm{M} \mathrm{CV} \mathrm{PU,} \mathrm{the} \mathrm{peak} \mathrm{is} \mathrm{at} \sim 645$ $\mathrm{nm}$ which is very similar to that observed when CV at $10 \mu \mathrm{M}$ is bound to serum albumin in aqueous solution. ${ }^{46}$ This red-shift effect is concentration-dependent and is ascribed to absorption of the shorter wavelength portion of the CV emission spectrum which suppresses emission nearer $600 \mathrm{~nm}$ and leads to the apparent red-shift. When both QD and CV (50 $\mu \mathrm{M})$ are present, the red-shift appears to be lower with peak emission at $667 \mathrm{~nm}$ compared to 686 $\mathrm{nm}$ for CV alone (Fig. 3C).

\section{Antibacterial Testing}

The antibacterial activity of QD and CV encapsulated polyurethane samples (QD + CV PU) was tested against methicillin-resistant isolate of Staphylococcus aureus, strain MRSA NCTC 13143 and a multi-drug resistant (MDR), carbapenemase-producing clinical strain of Escherichia coli (E. coli 1030). These bacterial strains were selected as representative multi-drug resistant (MDR) nosocomial Gram-positive and Gram-negative pathogens, respectively. ${ }^{47}$ The antibacterial activity of solvent-treated polyurethane (control PU), CV encapsulated polyurethane (CV PU) and QD encapsulated polyurethane (QD PU) was also tested and compared to QD + CV PU substrates.

Figure 4A shows the bactericidal activity of the various polymers against MRSA NCTC 13143 in the dark and following $1 \mathrm{~h}$ of exposure to a white light source with an average light intensity of $6600 \pm 990$ lux. Following $1 \mathrm{~h}$ of incubation in the dark, neither the control PU nor the QD PU samples showed significant kill of MRSA, whereas the CV and QD + CV PU samples showed a $1.2 \log _{10}$ and $0.7 \log _{10}$ reduction in bacterial numbers, respectively. Limited CV dark toxicity has been previously observed in our previous study on a laboratory strain of $S$. aureus which also used polyurethane substrates. ${ }^{38}$ Exposure to white light for a period of $1 \mathrm{~h}$ also did not result in any bactericidal activity from the control PU or QD PU but the addition of CV alone (CV PU) produced a $2.7 \log _{10}$ reduction in bacterial numbers. Furthermore, the combination of quantum dot and CV (QD + CV PU) caused the greatest kill of MRSA with numbers reduced by $3.7 \log _{10}(99.98 \% ; P=0.027$ compared to CV PU), following exposure to white light for just $1 \mathrm{~h}$.

(A)

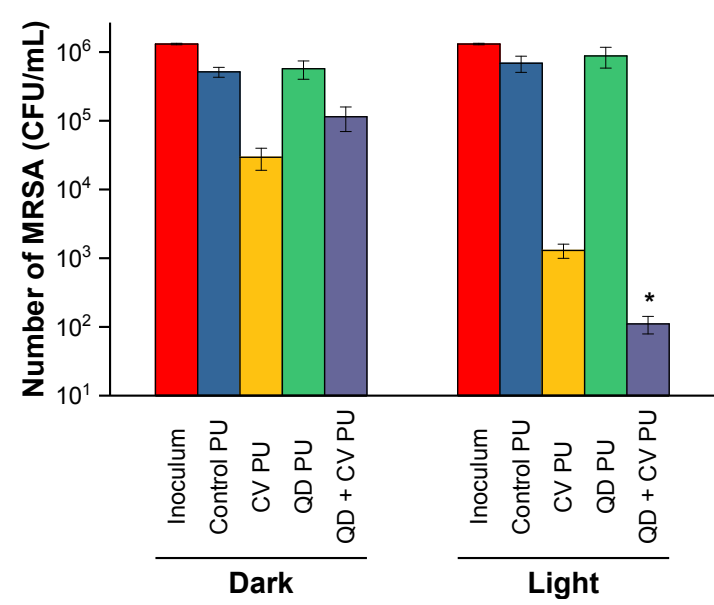

(B)

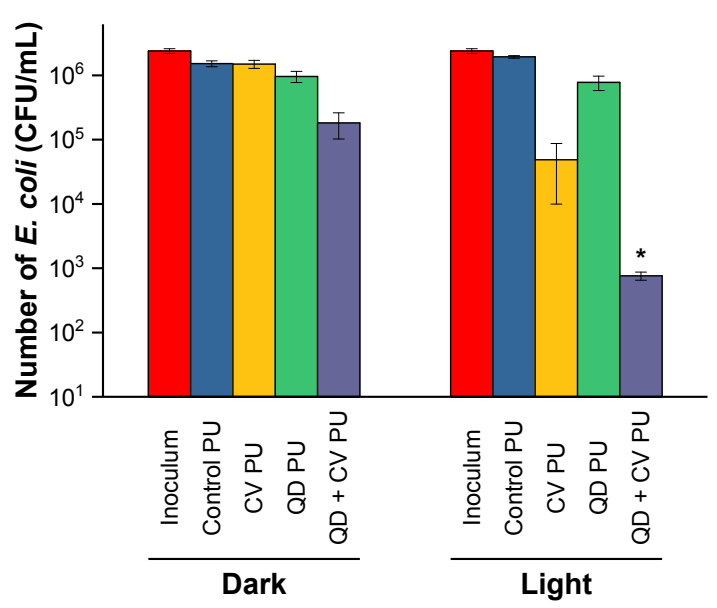

Figure 4. (A) Viable counts of MRSA NCTC 13143 on unmodified and modified polyurethane substrates incubated at $20^{\circ} \mathrm{C}$ under dark conditions and under white light (6000 lux \pm 990 lux) for $1 \mathrm{~h}$; (B) Viable counts of E. coli 1030 on unmodified and modified polyurethane substrates incubated at $20^{\circ} \mathrm{C}$ under 
dark conditions and under white light (6000 lux \pm 990 lux) for $4 \mathrm{~h}$. Concentrations of swelling solutions (made in 1:1 Cy/DCM solvent) used to modify PU: QDs $-1 \mathrm{mg} / \mathrm{mL} ; \mathrm{CV}-500 \mu \mathrm{M}$. (* indicates significance is $p<0.05$ compared to CV PU)

The photo-bactericidal activity of the polyurethane substrates was tested against MDR E. coli 1030, under the same conditions but for an extended period. As a Gram-negative bacterium, $E$. coli has a double membrane, consisting of both a cytoplasmic membrane and an outer membrane containing lipopolysaccharide which presumably accounts for the longer exposure time required for bactericidal activity. This contrasts with MRSA NCTC 13143, a Gram-positive bacterium, which possesses only a single cell membrane, allowing effective kill in a shorter period of time..$^{48}$ Figure 4B shows the activity of the samples after $4 \mathrm{~h}$ of incubation in the dark. Only the QD + CV PU substrate showed activity in the dark, inducing a $0.9 \log _{10}$ reduction in E. coli numbers after $4 \mathrm{~h}$ in the dark. White light illumination for a period of $4 \mathrm{~h}$ resulted in no significant bactericidal activity on the control PU material as expected, a $1.6 \log _{10}$ reduction in bacterial numbers was observed with CV PU, and a $0.4 \log _{10}$ reduction with QD PU. QD + CV PU showed increased bactericidal activity compared to CV PU alone, resulting in a $3.4 \log _{10}$ reduction in bacterial numbers following light exposure for $4 \mathrm{~h}(\mathrm{P}=0.019$ compared to $\mathrm{CV}$ $\mathrm{PU})$. Comparison of the fractional viabilities (i.e. the surviving fractions) shows that the combination of QDs with CV was synergistic for all strains tested (Table S1).

The potential of QDs in suspension to act as potent bactericidal light-activated agents has been studied previously. For instance, Ristic and co-workers reported the treatment of a clinical isolate of MRSA and reference strain E. coli ATCC 25922 with electrochemically produced graphene QDs in phosphate buffered saline (PBS). Following exposure of bacteria to $200 \mathrm{mg} / \mathrm{mL}$ of graphene QDs and irradiation with blue light $(465-475 \mathrm{~nm})$ for $15 \mathrm{~min}, E$. coli numbers were reduced by $80 \%$ and $S$. aureus numbers by about $95 \% .{ }^{26}$ Similarly, Courtney et al. prepared green-emitting cadmium telluride (CdTe) QDs directly prepared in aqueous media to which populations of MDR bacteria were exposed with and without visible light. In the presence of $100 \mathrm{nM} \mathrm{CdTe}$ and light for $8 \mathrm{~h}$, the growth of patient isolates of MRSA and Klebsiella pneumoniae were reduced by $29 \%$ and $59 \%$ respectively. Under the same conditions, a clinical isolate of MDR Salmonella typhimurium showed $56 \%$ growth inhibition and two MDR $E$. coli isolates had $83 \%$ and $64 \%$ growth inhibition, respectively. ${ }^{23}$ They demonstrated that superoxide was generated by photoexcitation of the QDs, and attributed the bactericidal activity to oxidative damage by this species and other ROS generated via the Fenton process.

However, in our study, polymer samples with QD alone were ineffective using the light doses (6000 lux) employed which we assume is due in part to limited uptake into the polymer via the simple swell-encapsulation technique, ${ }^{49}$ coupled with the use of visible light illumination where QD absorption is weaker compared to UV illumination (Figure 2, yellow line). A further key limiting factor in the use of QD-only doped polymer is that cumulative singlet oxygen and, more critically, superoxide generation by polymer-bound QDs is likely to be low in contrast to studies where QDs interact directly with cells. Yaghini et al. have shown that superoxide generation efficiency is significantly higher when QDs can interact with the important biomolecule nicotinamide adenine dinucleotide (NADH) which is present at high concentrations in cells via a Type 1 process. $^{30}$

The significant enhancement in bactericidal activity in the presence of CV shows that the addition of a photosensitising dye is essential for lethal photosensitisation in polymers, since QDs on their own elicit little to no antibacterial effect in polyurethane at these light doses. The use of CV only did elicit bacterial kill, as we have observed in other studies, ${ }^{14,38}$ but significantly less than the combination of the QD and CV. The observed enhancement of the intrinsic antibacterial properties of CV by the introduction of QDs suggests the occurrence of additional sources of ROS generation via interaction between the QD and CV via Förster resonance 
energy transfer (FRET) and/or photoelectron transfer (PET) involving the transfer of an electron or hole in this system.

\section{Photophysical studies}

(A)

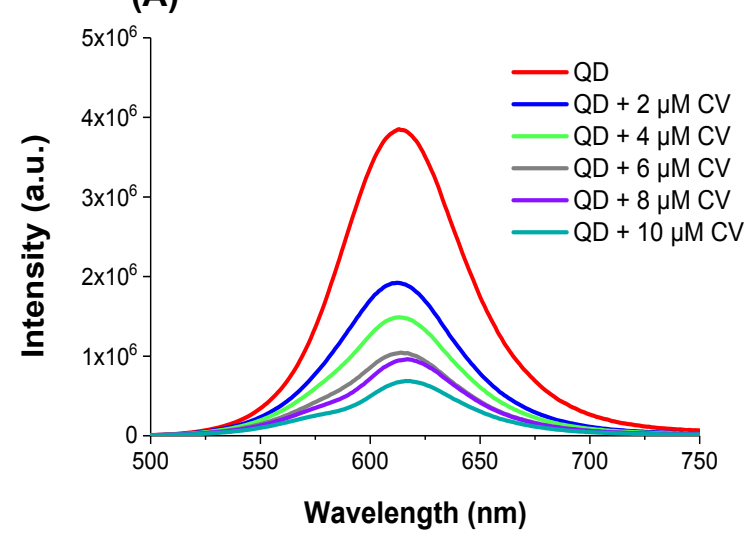

(B)

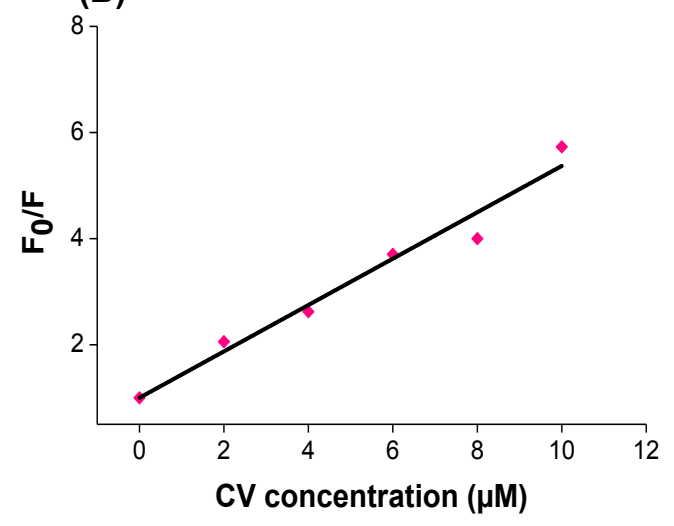

Figure 5. (A) Steady-state photoluminescence spectra of pure QDs and QD-CV combinations with increasing CV concentration in 1:1 Cy/DCM solvent system. Spectra from each sample was recorded following excitation at $400 \mathrm{~nm}$ where $\mathrm{CV}$ has negligible absorbance. Concentration of QDs stayed constant at $0.02 \mathrm{mg} / \mathrm{mL}$ as CV concentration was increased. (B) Stern-Volmer plot (at $620 \mathrm{~nm}, \lambda_{\mathrm{ex}}=400$ $\mathrm{nm}$ ) showing the relative changes in the emission intensity of the QDs as a function of CV dye concentration.

As a cationic dye in an aprotic solvent system, CV will be electrostatically attracted to the polar surface of QDs, which is capped with electronegative ligands, to form electrostatically bound complexes, which will enable the occurrence of short-range energy transfer interactions to take place. ${ }^{50-51} \mathrm{In}$ order to ascertain whether a QD-CV complex can function as a donoracceptor hybrid for FRET, the spectral overlap of CV with the QD must be demonstrated. As shown in figure 2, there is spectral overlap between the emission spectrum of the QDs and absorption spectrum of $\mathrm{CV}$ which, in principle, should enable the occurrence of FRET between donor QDs and the acceptor dye when blue light excitation employed. The overlap integral $J(\lambda)$, a quantitative measure of the degree of spectral overlap between the donor emission and the acceptor absorption was calculated using the equation:

$J(\lambda)=\int_{0}^{\infty} f_{D}(\lambda) \varepsilon_{A}(\lambda) \lambda^{4} d \lambda$

where $f_{D}(\lambda)$ is the dimensionless fluorescence intensity of the donor in the absence of the acceptor, $\varepsilon_{A}(\lambda)$ the extinction coefficient of the acceptor, in units of $\mathrm{M}^{-1} \mathrm{~cm}^{-1}$ and $\lambda$ is the wavelength in nanometres. ${ }^{52} J(\lambda)$ of the QD-CV complex was calculated to be $1.35 \times 10^{14}$ $\mathrm{nm}^{4} \mathrm{M}^{-1} \mathrm{~cm}^{-1}$, a relatively high overlap integral in accordance with the good spectral overlap of the QD-CV pair, which would favour non-radiative energy transfer from photoexcited QD donor to ground state photosensitiser acceptor. ${ }^{53}$

The corresponding Förster distance $\left(R_{0}\right)$, the distance at which the FRET efficiency is $50 \%$, was then derived as $3.4 \mathrm{~nm}$, using the equation:

$R_{0}=0.0211\left(\kappa^{2} \phi n^{-4} J(\lambda)\right)^{1 / 6}$

where $k$ is the orientation faction $\left(\kappa^{2}=2 / 3\right), \Phi$ is the quantum dot fluorescence quantum yield, $\mathrm{n}$ the refractive index and $J(\lambda)$ the spectral overlap. ${ }^{52}$ 
Steady-state and time-resolved QD photoluminescence studies were carried out to investigate the nature of the interaction between QDs and CV and the mechanism of the photoactivity of QD and CV mixtures in solution and the polymer. A fixed concentration of QDs and increasing concentrations of CV were prepared in solution and excited at $400 \mathrm{~nm}$, where the QD is excited but CV has negligible absorbance. Results in solution show a significant decrease in QD emission but no apparent CV fluorescence at longer wavelengths (Figure 5). CV absorption of the QD emission at $620 \mathrm{~nm}$ and longer wavelengths cannot account for the large decrease in intensity over this range of CV concentrations. ${ }^{43}$ It has been previously reported that the FRET efficiency increases when the number of acceptors per QD increases. We also observed that the decrease in QDs emission intensity was dependent on the CV/QD ratio and with increasing CV/QDs ratio, the QDs emission decreased progressively. If FRET occurs, then an increase in the acceptor emission should be observed. The apparent absence of the CV emission may however simply be due to the low fluorescence efficiency of the CV even when bound to the QD which has a very high quantum yield in comparison. ${ }^{46}$ Alternatively, photoinduced electron transfer (PET) between QDs and CV may take place, as found for a $\mathrm{CdSe} / \mathrm{Zn} \mathrm{QD} /$ fluorescein electrostatically bound complex in heptane, ${ }^{51}$ resulting in generation of the semi-reduced $\mathrm{CV}$ radical with different spectral properties, as discussed later in detail.

The static or dynamic nature of the quenching processes was measured using Stern-Volmer plots. The decrease in photoluminescence/fluorescence intensity is described by the SternVolmer (SV) relationship in equation (3)

$$
\begin{aligned}
& F_{0} /_{F}=1+K_{S V}[Q] \\
& K_{S V}[Q]=k_{q} \times \tau_{0}
\end{aligned}
$$

where $F_{0}$ and $F$ are the fluorescence intensities in the absence and presence of acceptor respectively, $[Q]$ the quencher concentration and $K_{S V}$ the Stern-Volmer quenching constant.

Dynamic quenching involves deactivation of the QD exciton upon contact with the quencher, in this case the $\mathrm{CV}$ molecules present in solution. Conversely, in static quenching, a complex is formed between the QD and the quencher, which in this case would arise from adsorption of the CV onto the QD surface, and the photoluminescence quenching can arise from either energy or electron transfer processes. ${ }^{52} 54$ The SV plot $\left(F_{0} / F\right.$ against [Q] $)$ at $620 \mathrm{~nm}\left(\lambda_{e x}=400\right.$ $\mathrm{nm}$ ) for QDs donor with CV acceptor in a solvent system of 1:1 cyclohexane/dichloromethane is shown in Fig. 5B. The linear SV relationship with a slope, $K_{S V}$ of $4.4 \times 10^{5} \mathrm{M}^{-1}$ is indicative of a single class of fluorophores, all equally accessible to the quencher. This was confirmed by estimating the bimolecular quenching constant $\left(k_{q}\right)$ from equation (4) using the slope obtained from the Stern-Volmer analysis $\left(K_{S V}[Q]\right)$ and the amplitude weighted lifetime of the donor QD $\left(\tau_{0}\right)$ in solution, as set out in equation 5 , which was measured to be 46 ns using time-correlated single photon counting. The large value of quenching constant $\left(k_{q}\right)$ of $8.91 \mathrm{x}$ $10^{12} \mathrm{M}^{-1} \mathrm{~s}^{-1}$ observed on addition of $\mathrm{CV}$, is two orders of magnitude higher than the diffusion controlled limit for bimolecular reactions, and shows that the interaction was predominantly static in nature with CV bound to the QD surface. ${ }^{55}$

From these studies, we conclude that direct interaction between the QDs and CV takes place in solution via adsorption of CV onto the QD surface which will enable occurrence of FRET and/or PET. In the strongly light-scattering polymer samples, quantitative absorption spectroscopy studies are difficult unless the samples are very thin unlike the samples here. The spectra shown in Figure 3C indicate that QD photoluminescence quenching in the presence of $\mathrm{CV}$ does take place in the polymer samples but to confirm occurrence of quenching FRET or PET, time-resolved fluorescence data was acquired by QDs donor lifetime 
measurements. Analysis of time-resolved fluorescence data can discriminate against apparent donor emission intensity attenuation due to facile reabsorption effects. For example, in a previous study in aqueous solutions, we observed that the mixtures of PEGylated $\mathrm{CdSe} / \mathrm{ZnS}$ QDs with toluidine blue O could enhance bacterial kill following visible illumination. However, QD time-resolved emission measurements found no evidence for FRET interaction and the associated lifetime shortening, and the enhanced activity was attributed to reabsorption of QD emission by the dye. ${ }^{25}$

In this study, excitation of the QDs was carried out at $405 \mathrm{~nm}$ where QD absorption is strong whereas CV absorption is very weak. The QD lifetime is also long compared to CV fluorescence lifetime which is $<5 \mathrm{~ns}$ in viscous solvents or when bound to protein. ${ }^{46,56}$

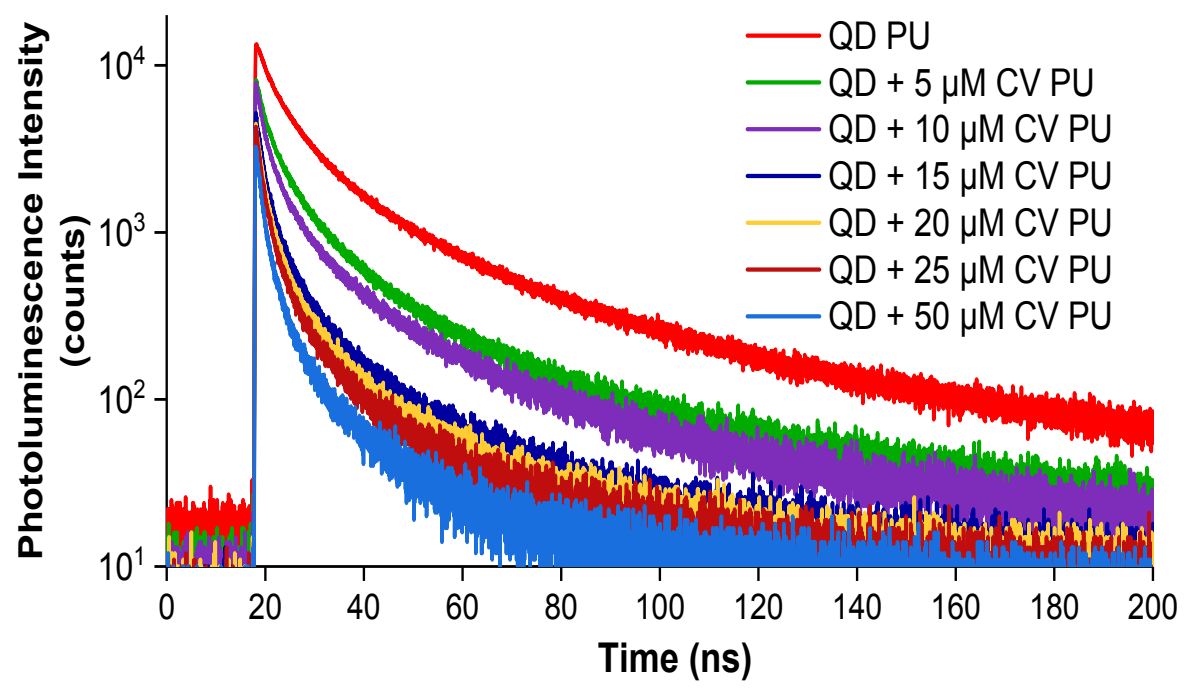

Figure 6. Logarithmic plots of time-resolved photoluminescence measurements of pure QDs and QDCV combination embedded in polyurethane via swell-encapsulation-shrink. Time-resolved QD emission lifetimes at $620 \mathrm{~nm}$ were measured after swelling polyurethane in solutions with final concentrations of $0.02 \mathrm{mg} / \mathrm{mL}$ QDs (blue line), $0.02 \mathrm{mg} / \mathrm{mL}$ QDs and $5 \mu \mathrm{M} \mathrm{CV}$ (red line), $0.02 \mathrm{mg} / \mathrm{mL}$ QDs and $10 \mu \mathrm{M} \mathrm{CV}$ (green line), $0.02 \mathrm{mg} / \mathrm{mL}$ QDs and $15 \mu \mathrm{M} \mathrm{CV}$ (purple line), $0.02 \mathrm{mg} / \mathrm{mL}$ QDs and $20 \mu \mathrm{M} \mathrm{CV}$ (light blue), $0.02 \mathrm{mg} / \mathrm{mL}$ QDs and $25 \mu \mathrm{M} \mathrm{CV}$ (orange line), $0.02 \mathrm{mg} / \mathrm{mL}$ and $50 \mu \mathrm{M} \mathrm{CV}$ (grey line).

Measurements were taken of the QD photoluminescence decay when only QDs were embedded in polyurethane as well as the QD decay when QDs with varying concentrations of $\mathrm{CV}$ were incorporated into polyurethane. Subsequently, the donor exciton lifetime was derived by fitting the time-resolved photoluminescence intensity $\left(I_{t}\right)$ of the $\mathrm{QD}$ to a bi-exponential decay of the form,

$I_{t}=A_{1} e^{\left(-t / \tau_{1}\right)}+A_{2} e^{\left(-t / \tau_{2}\right)}$

where $A$ and $\tau$ are the fractional amplitude and emission lifetime respectively. ${ }^{57}$

It was observed that the QD emission lifetime in the presence of CV $\left(\tau_{D A}\right)$ was significantly shorter than the QD emission lifetime in the absence of CV $\left(\tau_{D A}\right)$. The exciton photoluminescence lifetime progressively shortened with the introduction of increasingly higher concentrations of the dye into the polymer (Fig. 6). In the absence of the photosensitiser, the mean fractional amplitude weighted lifetime of QDs was found to be $\tau_{D}=$ $32.0 \mathrm{~ns}$ which was significantly reduced to $\tau_{D A}=6.8 \mathrm{~ns}$ in the presence of $50 \mu \mathrm{M} \mathrm{CV}$. 
If the quenching is due to FRET, the FRET efficiency $(E)$ of donor/acceptor complexes in polymer can be derived from the time-resolved fluorescence measurements, using the equation:

$E=1-\frac{\tau_{D A}}{\tau_{D}}$

where $\tau_{D A}$ and $\tau_{D}$ are the average fluorescence lifetime (amplitude weighted) of the donor in the presence and absence of the acceptor, respectively in polyurethane..$^{57-58}$

Table 1 displays the FRET efficiencies of the QD-CV with increasing CV concentration. At the highest crystal violet concentration, the FRET efficiency was calculated as $79 \%$. Note however that the $\mathrm{CV}$ concentration given is that of the dipping solution and we assume an approximately linear dependence between these values and the polymer uptake. We also assume that the use of the single-stage swell-encapsulation loading with both agents combined resulted in uptake of intact QD-CV complexes and CV residing in the matrix but in close proximity to the QDs.

It is important to note that the lifetime quenching observed is also consistent with the occurrence of PET therefore on the basis of these studies alone, we cannot be certain that FRET does take place in addition to PET. ${ }^{54}$ However since the PET mechanism should not lead to singlet oxygen generation, any ${ }^{1} \mathrm{O}_{2}$ produced by the QD-CV polymers would give distinct evidence of FRET. Therefore, in addition to steady-state and time-resolved fluorescence studies, the generation of singlet oxygen by the polymer samples was examined.

Table 1 Mean fractional amplitude weighted QD emission lifetimes and calculated FRET efficiencies of pure QD and QD-CV complexes with increasing CV concentrations (in the swell-encapsulation medium) when incorporated into polyurethane via the swell-encapsulation-shrink method

\begin{tabular}{|lll|}
\hline $\begin{array}{l}\text { CV } \\
\text { Concentration } \\
(\boldsymbol{\mu M})\end{array}$ & Mean $\boldsymbol{\tau}$ (ns) & $\begin{array}{l}\text { FRET Efficiency } \\
(\%)\end{array}$ \\
\hline 0 & 32.0 & 0 \\
\hline 5 & 28.2 & 12 \\
\hline 10 & 21.6 & 33 \\
\hline 15 & 17.0 & 47 \\
\hline 20 & 14.7 & 54 \\
\hline 25 & 7.8 & 75 \\
\hline 50 & 6.8 & 79 \\
\hline
\end{tabular}

Singlet Oxygen Phosphorescence Studies

The generation of ${ }^{1} \mathrm{O}_{2}$ in the polymer was investigated using time-resolved near-IR detection based on the singlet oxygen phosphorescence at $1270 \mathrm{~nm}$. We have previously shown that CV alone in silicone can generate singlet oxygen using excitation at $532 \mathrm{~nm} .{ }^{59}$ However, in aqueous solution, the efficiency of ${ }^{1} \mathrm{O}_{2}$ is very low owing to deactivation of the CV excited singlet state by the molecular rotor effect. In viscous solvents or the polymeric microenvironment, this effect is inhibited which permits ${ }^{1} \mathrm{O}_{2}$ generation. 


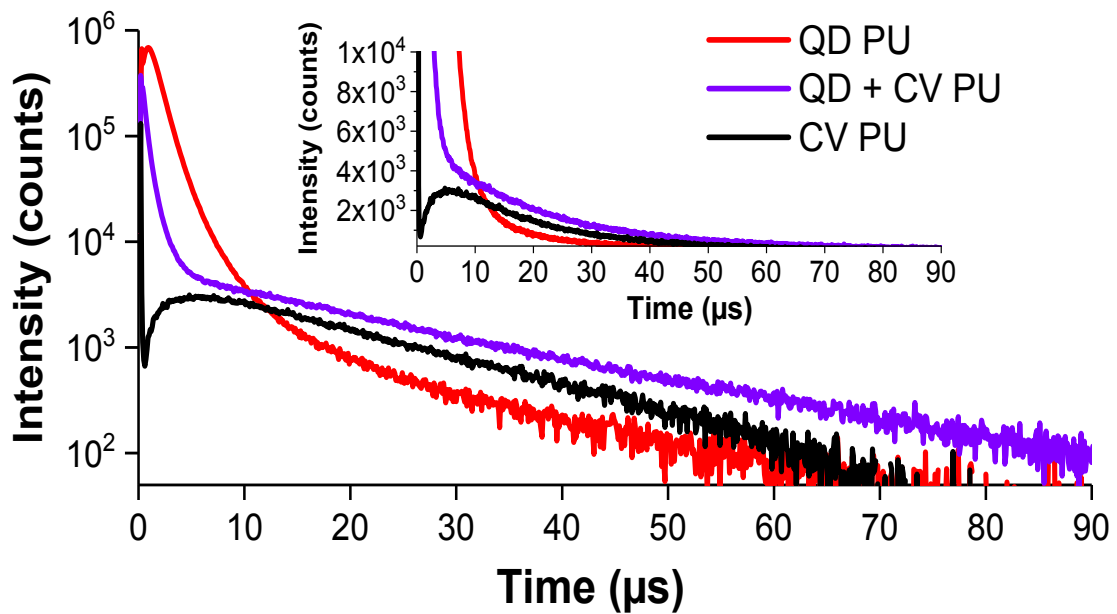

Figure 7 Logarithmic time-resolved phosphorescence singlet oxygen recorded at $1270 \mathrm{~nm}$ following pulsed laser irradiation of modified polyurethane containing CV only (black line), QD only (red line and a combination of QD and CV (purple line). (Concentrations: QDs - $1 \mathrm{mg} / \mathrm{mL}$; CV $-50 \mu \mathrm{M} \mathrm{CV}$ ). The traces have been subtracted from the control (untreated polyurethane). Inset: Linear scale plot over the same time range

Figure 7 shows traces recorded for samples containing CV alone, QD and CV with QD using similar concentrations that are used for the antimicrobial assays. Logarithmic intensity plots are shown since this allows the differences in quasi-monoexponential decay lifetimes to be more readily discerned. For the QD alone trace, a relatively short-lived high intensity signal is observed, lasting $\sim 10 \mu \mathrm{s}$. This signal is ascribed to the near-IR tail of the QD photoluminescence. ${ }^{60}$ Although this signal will be very weak, the singlet oxygen phosphorescence is also very weak given the very low quantum yield. Direct generation of singlet oxygen by photoexcitation of indium-based QDs is energetically unfavourable although direct generation of superoxide may be possible, as discussed later. ${ }^{27}$ We have previously shown for CdS-based QDs that no direct singlet oxygen generation could be observed with an upper limit to the quantum yield estimated as $<0.003 .{ }^{30}$ Using spin-trap electron paramagnetic resonance (EPR) spectroscopy and reporter assays, Chibli et. al. found no evidence for the generation of singlet oxygen in indium phosphide based QDs. ${ }^{27}$ For CV alone, a characteristic initial increase followed by a near-exponential decay is observed, in agreement with our previous study ${ }^{59}$ using $\mathrm{CV}$ and other dyes such as methylene blue (MB). ${ }^{61}$ The initial rise in signal is governed by the rate of quenching of the $\mathrm{CV}$ triplet state to the ground state by oxygen to generate singlet oxygen in the polymer matrix. The subsequent decay in signal is governed by the rate of quenching of the singlet oxygen phosphorescence by the polymer matrix. The lifetime of singlet oxygen in the polyurethane where only $\mathrm{CV}$ was present, derived by fitting the signal to a mono-exponential decay, was determined to be 36 $\mu \mathrm{s}$. This value is similar to that observed for CV embedded in silicone polymer ( $40 \mu \mathrm{s}) .{ }^{59}$ The decay kinetics were not affected by the $\mathrm{CV}$ concentration within the ranges used. There is also a very short spike in signal at $t=0$ which is due to CV fluorescence. The presence of the QD with $\mathrm{CV}$ resulted in a major change in the initial time-dependence with the rise-time seen for $\mathrm{CV}$ alone replaced by an intense short-lived decay lasting $\sim 3 \mu$ s followed by a decay with a lifetime that closely matches that observed for CV alone which is consistent with the assumption that the same decay kinetics for singlet oxygen should apply for each type of polymer sample. However, the key observation from this comparative study is that the overall intensity of the singlet oxygen signal with the QD/CV combination was higher than for CV alone. 
The short but rapid decay in signal seen at early times $<10 \mu$ s in the presence of CV matches that observed in our previous QD FRET studies ${ }^{60}$ and is consistent with the QD donor photoluminescence being quenched by the CV. As can be seen from the logarithmic decay dependence, the quenched QD photoluminescence lifetime in the decay profile of QD + CV PU is considerably shorter than that for in the absence of CV (QD PU). Use of a lower concentration of $\mathrm{CV}$ of $5 \mu \mathrm{M}$ (not shown) resulted in a slower decay of the QD photoluminescence, which is consistent with the quenching mechanism. We can therefore conclude that singlet oxygen is generated in the polymer both by $\mathrm{CV}$ alone and by the interaction of $\mathrm{QD}$ and $\mathrm{CV}$. In the aforementioned solution phase studies, the driving force for QD/CV complex formation would be electrostatic attraction of the cationic CV to the polar QD surface. In the polymer matrix however, a proportion of the CV may also reside in the matrix in close proximity to the QDs but not bound at the QD surface. Since the Förster distance is estimated at $3.4 \mathrm{~nm}$, FRET would be viable for CV that is physically separated from the QD. A degree of spatial separation of the CV from the QDs in the polymer may also enable greater partitioning of the interaction mechanism to FRET over PET which would be favoured by the shorter range interaction when CV is surface-adsorbed. CV located further away from the QD beyond the energy transfer range could reabsorb the QD emission in a non-FRET process, in an analogous mechanism proposed by Narband et al. In their study of CdSe/ZnS QDs mixed with toluidine blue $O$ in aqueous solution, bactericidal activity was ascribed to facile absorption of QD emission by the photosensitiser. ${ }^{25}$ In the present study however, the QD emission is so strongly quenched by FRET or electron transfer (discussed below in more detail) that a reabsorption mechanism can be precluded. A further point to take into consideration is that the diffusion pathlength of the singlet oxygen in the polymer will be short $(<1 \text { micron })^{59}$ thus the active zone for ROS generation at the surface is shallow. This therefore severely limits the pathlength over which such reabsorption processes can take place.

\section{Superoxide Generation \& Inhibition Assay}

In addition to resonant energy transfer, it is also possible that a PET process can take place between the QD and CV when in close proximity. Photoexcited indium phosphide QDs are capable of reducing oxygen to superoxide ${ }^{27}$ and the reduction potential of the $\mathrm{O}_{2} / \mathrm{O}_{2}{ }^{-}$redox couple is $-0.18 \mathrm{~V}$ vs. NHE (normal hydrogen electrode) in aqueous solution (equation 7) which is close to that of $\mathrm{CV}^{+} / \mathrm{CV}^{\bullet}$ reduction potential at $-0.36 \mathrm{~V}$ (equation (8)). ${ }^{62-63} \mathrm{CV}$ is a good electron acceptor and photoreduction of CV by the QDs to generate the semi-reduced $\mathrm{CV}$ radical is likely to occur given the similar potentials. By extrapolation of excited-state redox potentials estimated for other indium-based QDs emitting at comparable wavelengths to the QDs used here, reduction of CV should be energetically feasible: the valence band reduction potential for InP quantum dots is estimated as $0.95 \mathrm{~V} 27,64$. This assumes that effects of binding and the different solvent on redox potentials are minimal.

The CV radical generated by PET from the QD can then interact with molecular oxygen to generate superoxide by electron transfer which should be energetically possible due to the positive redox potential $(+0.18 \mathrm{~V})$ of the reaction (equation $(9))$.

$$
\begin{array}{ll}
\mathrm{CV}^{+}+e^{-} \rightarrow \mathrm{CV}^{.} & -0.36 \mathrm{~V} \\
\mathrm{O}_{2}+e^{-} \rightarrow \mathrm{O}_{2}^{-} . & -0.18 \mathrm{~V} \\
\mathrm{CV}+\mathrm{O}_{2} \rightarrow \mathrm{CV}^{+}+\mathrm{O}_{2}^{-.} & +0.18 \mathrm{~V}
\end{array}
$$

Experimental evidence for this Type 1 process in aqueous solution has been obtained by detection of superoxide using electron paramagnetic resonance (EPR) spectroscopy following illumination of solutions of crystal violet containing the electron donor, $\mathrm{NADH}$, which donates an electron to the excited CV triplet state to form the CV radical. ${ }^{65-66}$ In the study by Fischer 
et al., micellar solutions of Triton-X were employed. ${ }^{66}$ We have to assume that this reaction scheme is also possible in the polymer matrix. However, the occurrence of Type 1 processes following illumination when $\mathrm{CV}$ is incubated directly with bacteria (where the $\mathrm{CV}$ is presumably bound with substrates and can interact with electron donors) lends support to the scheme we propose, whereby superoxide is generated via interaction of oxygen with the semi-reduced $\mathrm{CV}$ radical formed by photoreduction. ${ }^{46,67}$

We also need to consider the effects of direct photoexcitation of CV to its singlet state in the QD/CV complex. Since CV emission occurs at longer wavelengths than the QD absorption there is little spectral overlap to enable FRET to take place with the CV acting as the donor to the QD. FRET would also have to compete with the rapid internal non-radiative pathways of excited singlet CV state. Alternatively, electron transfer may be possible since spectral overlap is not required, and in this case the QD may act as an electron donor to the CV in its excited singlet or triplet state to form the CV radical thereby leading to superoxide formation in an analogous mechanism to aforementioned reaction where NADH acts as an electron donor to photoexcited CV. The CV triplet state is likely to act as the acceptor since it is longer lived than the excited singlet state. Since CV absorbs within the QD absorption band it would not be possible to excite $\mathrm{CV}$ alone therefore this process would be more difficult to discern experimentally. Instead of the QDs the polymer matrix, which will contain unreacted monomer, may also be able to promote a Type 1 process, and contribute to the cytotoxicity observed with CV alone. Photoexcitation of dimers of CV may also act as a source of superoxide via electron-transfer reactions as postulated previously for MB-doped polymer substrates. ${ }^{61}$ Direct generation of superoxide by electron transfer to oxygen by the CV triplet state has been postulated by Reszka et al. and Fischer et al. ${ }^{65-66}$ but was confirmed by Brezova and colleagues who demonstrated photogeneration of superoxide directly from CV in aprotic solvents using EPR. ${ }^{68}$ Therefore superoxide should be generated in illuminated polymer samples with CV alone and CV with QDs, and then be able to diffuse into the surrounding solution containing the bacteria. This mechanism is distinct from treating bacteria incubated in solutions containing a photosensitiser where the sensitiser can interact directly with the bacteria and exert photooxidative damage after internalisation or via electron or hydrogen abstraction reactions mediated by the sensitiser triplet state in contact with the membrane. ${ }^{69}$ The latter mechanism is unlikely to be effective for bactericidal polymer systems unless the polymer is designed specifically to release the sensitiser.

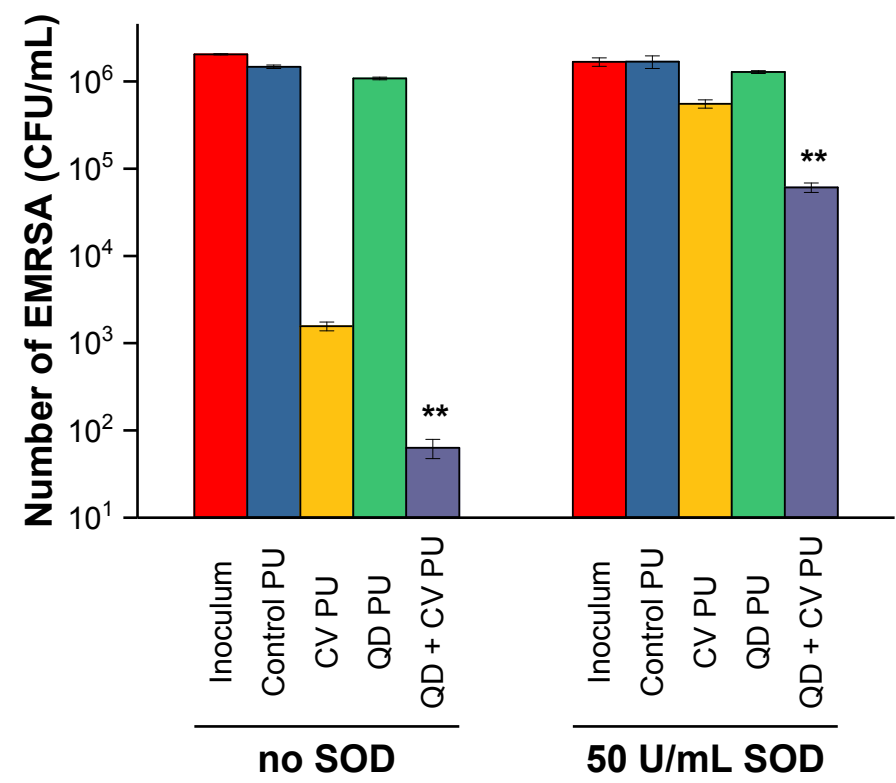

Figure 8 Viable counts of EMRSA 4742 on unmodified and modified polyurethane polymers tested under standard conditions $\left(20^{\circ} \mathrm{C}, 1 \mathrm{~h}\right.$ white light treatment at 6000 lux) without SOD (left) and in the presence of $50 \mathrm{U} / \mathrm{mL}$ SOD (same temperature and lighting conditions as standard test) (right). 
Concentrations of swelling solutions (made in 1:1 Cy/DCM solvent) used to modify PU: QDs $-1 \mathrm{mg} / \mathrm{mL}$; $\mathrm{CV}-500 \mu \mathrm{M}$. (** indicates significance of $p<0.01$ compared to CV PU)

To assess the possible contribution of Type I electron transfer pathways, the bactericidal activity of the control PU, CV PU and QD + CV PU substrates were tested against EMRSA 4742 after $1 \mathrm{~h}$ of white light treatment, both under standard conditions as well as in the presence of superoxide dismutase (SOD). SOD acts as a scavenger of superoxide, a radical generated by PET as discussed above. After $1 \mathrm{~h}$ exposure to light, neither the control PU nor QD PU demonstrated any significant bactericidal activity, and there was no change in activity in the presence of $50 \mathrm{U} / \mathrm{mL}$ SOD. Conversely, CV PU and QD + CV PU bactericidal activities were significantly diminished in the presence of SOD. The antibacterial activity of CV PU and QD + CV PU substrates decreased by $\sim 2.5 \log _{10}$ (from $99 \%$ to $66.9 \%$ ) and $\sim 3 \log _{10}$ (from $99.996 \%$ to $96.4 \%$ ) respectively when $50 \mathrm{U} / \mathrm{mL}$ SOD was added (Fig. 8).

The effectiveness of SOD in inhibiting the potent kill of CV PU and QD + CV PU upon irradiation indicates that $\mathrm{O}_{2}^{-\bullet}$ is formed by the materials then subsequently scavenged before oxidative damage occurs to the bacteria. Although superoxide is relatively unreactive, superoxide can undergo dismutation in solution before reaching the bacteria to hydrogen peroxide, which is an effective bactericidal agent. Furthermore, the iron-catalysed Fenton process will result in generation of the highly reactive hydroxyl radical species. In this case inhibition of superoxide using superoxide dismutase will also inhibit these ROS which is consistent with our data in Figure 8. Thus, conversion of superoxide to more potent ROS can compensate for its lower activity. Since we know that ${ }^{1} \mathrm{O}_{2}$ is generated as well, we conclude that the synergistic enhancement in antibacterial activity of QDs combined with CV is due to the combined action of ROS generated by both Type I and Type II mechanisms. The range of mechanisms of ROS generation using QDs and CV is distinct to our previous work using $2 \mathrm{~nm}$ diameter gold nanoparticles or $\mathrm{ZnO}$ nanoparticles and crystal violet in polymer substrates. In those studies where the nanoparticles exhibit negligible absorption in the visible range, FRET interaction would not apply and electron transfer interactions are likely to have taken place only via excitation of the CV. ${ }^{14,38}$

\section{Conclusion}

This study is the first to investigate the antibacterial effects of heavy metal-free quantum dots when embedded in a clinically relevant polymer, in order to assess the practicality and effectiveness of quantum dots in reducing bacterial contamination of surfaces. We have shown that indium-based quantum dot nanoparticles and crystal violet can be embedded into polyurethane via the swell-encapsulation-shrink technique using a simple 'one-pot' dipping process. Characterisation of polymer samples showed good uptake of QD and dye without the use of expensive, chemical or mechanical deposition methods. Owing to their larger size, it is likely that the QDs reside near the surface whereas free CV will also be encapsulated deeper within the polymer since it can disperse more readily owing to its smaller size. ${ }^{49}$. Since the ROS have a limited diffusion distance within the polymer before reaching the surface, the localisation of the QDs nanoparticles near the surface should be advantageous for a synergistic effect mediated by ROS. ${ }^{14}$ These novel QD-CV polymer substrates demonstrated highly effective antimicrobial activity against clinical strains of both Gram-positive and Gramnegative bacteria. The combination of the QDs and crystal violet caused $99.98 \%$ kill of two strains of methicillin-resistant Staphylococcus aureus after $1 \mathrm{~h}$ and $99.96 \%$ kill in a carbapenemase-producing Escherichia coli strain in $4 \mathrm{~h}$ using broad-band visible illumination at 6000 lux. Steady-state and time-resolved QD fluorescence and singlet oxygen phosphorescence analysis confirmed that the QDs and CV can interact to promote generation of reactive oxygen species. Singlet oxygen Type II reactions enabled enhancement of crystal violet's intrinsic antimicrobial properties when combined with QDs, while antimicrobial tests in the presence of SOD indicated the involvement of Type I reactions for QD + CV PU substrates. The contribution from both processes amplifies QD + CV PU substrate activity, however 
further analysis will be required to evaluate which mechanism has a greater contribution to the photo-bactericidal activity of the materials.

The efficacy of these QD-CV polymer substrates that can harvest light across the visible spectrum, against multi-drug resistant bacteria demonstrates the potential of quantum dotphotosensitiser combinations for use in disinfecting hospital surfaces and devices. This therapeutic approach generates ROS that cause bacterial death by attacking multiple sites on the microorganisms, making the development of resistance unlikely. Thus, QD-CV polymers show promise assisting to decrease the risk of infections by reducing the levels of environmental contamination. The technique will also be applicable for other medically used polymers such as silicone.

\section{Supporting Information}

Fluorescence emission spectra of crystal violet-incorporated polyurethane and statistical analysis of bactericidal activity of materials

\section{Acknowledgements}

We acknowledge support from the Medical Research Council for the award of a CASE studentship (grant award MR/M015866/1) to support this work (EO).

\section{References}

1. Organisation for Economic Co-operation and Development (OECD), Health at a Glance:

Europe 2016: State of Health in the EU Cycle. OECD Publishing: 2016.

2. $\quad K$ Klevens, R. M.; Edwards, J. R.; Richards, C. L.; Horan, T. C.; Gaynes, R. P.; Pollock, D. A.; Cardo, D. M., Estimating Health Care-Associated Infections and Deaths in US Hospitals, 2002. Public Health Rep. 2007, 122 (2), 160-166.

3. Samanipour, A.; Dashti-Khavidaki, S.; Abbasi, M. R.; Abdollahi, A., Antibiotic Resistance Patterns of Microorganisms Isolated from Nephrology and Kidney Transplant Wards of a Referral Academic Hospital. J Res Pharm Pract 2016, 5 (1), 43-51.

4. Report on the Burden of Endemic Health Care-Associated Infection Worldwide. World Health Organization (WHO): 2011.

5. Collins, A., Preventing Health Care-Associated Infections. In Patient Safety and Quality: An Evidence-Based Handbook for Nurses., Agency for Healthcare Research and Quality (US): April 2008.

6. Wainwright, M.; Phoenix, D. A.; Marland, J.; Wareing, D. R. A.; Bolton, F. J., A Study of Photobactericidal Activity in the Phenothiazinium Series. FEMS Immunol. Med. Microbiol. 1997, 19 (1), 75-80.

7. Wainwright, M.; Crossley, K. B., Photosensitising Agents - Circumventing Resistance and Breaking Down Biofilms: A Review. Int. Biodeterior. Biodegrad. 2004, 53 (2), 119-126.

8. Demidova, T. N.; Hamblin, M. R., Photodynamic Inactivation of Bacillus Spores, Mediated by Phenothiazinium Dyes. Appl. Environ. Microbiol. 2005, 71 (11), 6918-6925.

9. Meisel, P.; Kocher, T., Photodynamic Therapy for Periodontal Diseases: State of the Art. J. Photochem. Photobiol. B-Biol. 2005, 79 (2), 159-170.

10. Saji, M.; Taguchi, S.; Uchiyama, K.; Osono, E.; Hayama, N.; Ohkuni, H., Efficacy of GentianViolet in the Eradication of Methicillin-Resistant Staphylococcus-Aureus from Skin-Lesions. J Hosp Infect 1995, 31 (3), 225-228.

11. Perni, S.; Piccirillo, C.; Pratten, J.; Prokopovich, P.; Chrzanowski, W.; Parkin, I. P.; Wilson, M., The Antimicrobial Properties of Light-Activated Polymers Containing Methylene Blue and Gold Nanoparticles. Biomaterials 2009, 30 (1), 89-93.

12. Perni, S.; Prokopovich, P.; Piccirillo, C.; Pratten, J.; Parkin, I. P.; Wilson, M., Toluidine BlueContaining Polymers Exhibit Potent Bactericidal Activity when Irradiated with Red Laser Light. J Mater Chem 2009, 19 (18), 2715-2723.

13. Noimark, S.; Dunnill, C. W.; Kay, C. W. M.; Perni, S.; Prokopovich, P.; Ismail, S.; Wilson, M.; Parkin, I. P., Incorporation of Methylene Blue and Nanogold into Polyvinyl Chloride Catheters; A New Approach for Light-Activated Disinfection of Surfaces. J Mater Chem 2012, 22 (30), 15388-15396. 
14. Noimark, S.; Bovis, M.; MacRobert, A. J.; Correia, A.; Allan, E.; Wilson, M.; Parkin, I. P., Photobactericidal Polymers; The Incorporation of Crystal Violet and Nanogold into Medical Grade Silicone. RSC Adv 2013, 3 (40), 18383-18394.

15. Dolmans, D. E.; Fukumura, D.; Jain, R. K., Photodynamic Therapy for Cancer. Nat Rev Cancer 2003, 3 (5), 380-387.

16. Alivisatos, A. P.; Gu, W. W.; Larabell, C., Quantum Dots as Cellular Probes. Annu. Rev. Biomed. Eng. 2005, 7, 55-76.

17. Samia, A. C. S.; Dayal, S.; Burda, C., Quantum Dot-Based Energy Transfer: Perspectives and Potential for Applications in Photodynamic Therapy. Photochem Photobiol 2006, 82 (3), $617-625$.

18. Gill, R.; Zayats, M.; Willner, I., Semiconductor Quantum Dots for Bioanalysis. Angew. Chem., Int. Ed. Eng. 2008, 47 (40), 7602-7625.

19. Yu, W. W.; Chang, E.; Drezek, R.; Colvin, V. L., Water-Soluble Quantum Dots for Biomedical Applications. Biochem. Biophys. Res. Commun. 2006, 348 (3), 781-786.

20. Resch-Genger, U.; Grabolle, M.; Cavaliere-Jaricot, S.; Nitschke, R.; Nann, T., Quantum Dots versus Organic Dyes as Fluorescent Labels. Nat Methods 2008, 5 (9), 763-775.

21. Xu, G. X.; Zeng, S. W.; Zhang, B. T.; Swihart, M. T.; Yong, K. T.; Prasad, P. N., New Generation Cadmium-Free Quantum Dots for Biophotonics and Nanomedicine. Chem Rev 2016, 116 (19), 12234-12327.

22. Yaghini, E.; Seifalian, A. M.; MacRobert, A. J., Quantum Dots and their Potential Biomedical Applications in Photosensitization for Photodynamic Therapy. Nanomedicine (Lond.) 2009, 4 (3), $353-$ 363.

23. Courtney, C. M.; Goodman, S. M.; McDaniel, J. A.; Madinger, N. E.; Chatterjee, A.; Nagpal, P., Photoexcited Quantum Dots for Killing Multidrug-Resistant Bacteria. Nat Mater 2016, 15 (5), 529 534.

24. Ipe, B. I.; Lehnig, M.; Niemeyer, C. M., On the Generation of Free Radical Species from Quantum Dots. Small 2005, 1 (7), 706-709.

25. Narband, N.; Mubarak, M.; Ready, D.; Parkin, I. P.; Nair, S. P.; Green, M. A.; Beeby, A.; Wilson, M., Quantum Dots as Enhancers of the Efficacy of Bacterial Lethal Photosensitization. Nanotechnology 2008, 19 (44).

26. Ristic, B. Z.; Milenkovic, M. M.; Dakic, I. R.; Todorovic-Markovic, B. M.; Milosavljevic, M. S.; Budimir, M. D.; Paunovic, V. G.; Dramicanin, M. D.; Markovic, Z. M.; Trajkovic, V. S., Photodynamic Antibacterial Effect of Graphene Quantum Dots. Biomaterials 2014, 35 (15), 4428-4435.

27. Chibli, H.; Carlini, L.; Park, S.; Dimitrijevic, N. M.; Nadeau, J. L., Cytotoxicity of InP/ZnS Quantum Dots Related to Reactive Oxygen Species Generation. Nanoscale 2011, 3 (6), 2552-2559. 28. Ge, J.; Lan, M.; Zhou, B.; Liu, W.; Guo, L.; Wang, H.; Jia, Q.; Niu, G.; Huang, X.; Zhou, H.; Meng, X.; Wang, P.; Lee, C. S.; Zhang, W.; Han, X., A Graphene Quantum Dot Photodynamic Therapy Agent with High Singlet Oxygen Generation. Nat Commun 2014, 5, 4596.

29. Ge, J. C.; Lan, M. H.; Liu, W. M.; Jia, Q. Y.; Guo, L.; Zhou, B. J.; Meng, X. M.; Niu, G. L.; Wang, P. F., Graphene Quantum Dots as Efficient, Metal-Free, Visible-Light-Active Photocatalysts. Sci China Mater 2016, 59 (1), 12-19.

30. Yaghini, E.; Pirker, K. F.; Kay, C. W. M.; Seifalian, A. M.; MacRobert, A. J., Quantification of Reactive Oxygen Species Generation by Photoexcitation of PEGylated Quantum Dots. Small 2014, $10(24), 5106-5115$.

31. Moeno, S.; Antunes, E.; Khene, S.; Litwinski, C.; Nyokong, T., The Effect of Substituents on the Photoinduced Energy Transfer between CdTe Quantum Dots and Mercapto Substituted Zinc Phthalocyanine Derivatives. Dalton T 2010, 39 (14), 3460-3471.

32. Charron, G.; Stuchinskaya, T.; Edwards, D. R.; Russell, D. A.; Nann, T., Insights into the Mechanism of Quantum Dot-Sensitized Singlet Oxygen Production for Photodynamic Therapy. J Phys Chem C 2012, 116 (16), 9334-9342.

33. Derfus, A. M.; Chan, W. C. W.; Bhatia, S. N., Probing the Cytotoxicity of Semiconductor Quantum Dots. Nano Lett 2004, 4 (1), 11-18.

34. Cho, S. J.; Maysinger, D.; Jain, M.; Roder, B.; Hackbarth, S.; Winnik, F. M., Long-Term Exposure to CdTe Quantum Dots Causes Functional Impairments in Live Cells. Langmuir 2007, 23 (4), 1974-1980.

35. Yaghini, E.; Turner, H. D.; Le Marois, A. M.; Suhling, K.; Naasani, I.; MacRobert, A. J., In vivo biodistribution studies and ex vivo lymph node imaging using heavy metal-free quantum dots.

Biomaterials 2016, 104, 182-191.

36. Yaghini, E.; Turner, H.; Pilling, A.; Naasani, I.; MacRobert, A. J., In Vivo Biodistribution and Toxicology Studies of Cadmium-Free Indium-Based Quantum Dot Nanoparticles in a Rat Model. Nanomedicine (Lond.) 2018, 14 (8), 2644-2655. 
38. Sehmi, S. K.; Noimark, S.; Bear, J. C.; Peveler, W. J.; Bovis, M.; Allan, E.; MacRobert, A. J.; Parkin, I. P., Lethal Photosensitisation of Staphylococcus aureus and Escherichia coli Using Crystal Violet and Zinc Oxide-Encapsulated Polyurethane. J Mater Chem B 2015, 3 (31), 6490-6500.

39. Maley, A. M.; Arbiser, J. L., Gentian Violet: A 19th Century Drug Re-Emerges in the 21st Century. Exp Dermatol 2013, 22 (12), 775-780.

40. Arbiser, J. L., Gentian Violet is Safe. J Am Acad Dermatol 2009, 61 (2), 359-359.

41. Berrios, R. L.; Arbiser, J. L., Effectiveness of Gentian Violet and Similar Products Commonly Used to Treat Pyodermas. Dermatol Clin 2011, 29 (1), 69-73.

42. Dimitrijevic, N. M.; Takahashi, K.; Jonah, C. D., Visible Absorption Spectra of Crystal Violet in Supercritical Ethane-Methanol Solution. J Supercrit Fluid 2002, 24 (2), 153-159.

43. Oliveira, C. S.; Branco, K. P.; Baptista, M. S.; Indig, G. L., Solvent and Concentration Effects on the Visible Spectra of Tri-Para-Dialkylamino-Substituted Triarylmethane Dyes in Liquid Solutions. Spectroc. Acta Pt. A-Molec. Biomolec. Spectr. 2002, 58 (13), 2971-2982.

44. Brey, L. A.; Schuster, G. B.; Drickamer, H. G., High-Pressure Studies of Effect of Viscosity on Fluorescence Efficiency in Crystal Violet and Auramine O. J Chem Phys 1977, 67 (6), 2648-2650.

45. Cremers, D. A.; Windsor, M. W., A Study of the Viscosity-Dependent Electronic Relaxation of Some Triphenylmethane Dyes Using Picosecond Flash-Photolysis. Chemical Physics Letters 1980, 71 (1), 27-32.

46. Baptista, M. S.; Indig, G. L., Effect of BSA Binding on Photophysical and Photochemical Properties of Triarylmethane Dyes. J Phys Chem B 1998, 102 (23), 4678-4688.

47. Global Priority List of Antibiotic-Resistant Bacteria to Guide Research, Discovery and Development of New Antibiotics; World Health Organization (WHO): 2017.

48. Silhavy, T. J.; Kahne, D.; Walker, S., The Bacterial Cell Envelope. Cold Spring Harb Perspect Biol 2010, 2 (5), a000414.

49. Crick, C. R.; Noimark, S.; Peveler, W. J.; Bear, J. C.; Ivanov, A. P.; Edel, J. B.; Parkin, I. P., Advanced Analysis of Nanoparticle Composites - A Means toward Increasing the Efficiency of Functional Materials. RSC Adv 2015, 5 (66), 53789-53795.

50. Devatha, G.; Roy, S.; Rao, A.; Mallick, A.; Basu, S.; Pillai, P. P., Electrostatically Driven Resonance Energy Transfer in "Cationic" Biocompatible Indium Phosphide Quantum Dots. Chem Sci 2017, 8 (5), 3879-3884.

51. Issac, A.; Jin, S. Y.; Lian, T. Q., Intermittent Electron Transfer Activity from Single CdSe/ZnS Quantum Dots. J. Am. Chem. Soc. 2008, 130 (34), 11280-11281.

52. Lakowicz, J. R., Principles of Fluorescence Spectroscopy. 3rd ed. ed.; New York : Springer: New York, 2006.

53. Medintz, I. L.; Mattoussi, H., Quantum Dot-Based Resonance Energy Transfer and its Growing Application in Biology. Phys Chem Chem Phys 2009, 11 (1), 17-45.

54. Murphy, C. B.; Zhang, Y.; Troxler, T.; Ferry, V.; Martin, J. J.; Jones, W. E., Probing Forster and Dexter Energy-Transfer Mechanisms in Fluorescent Conjugated Polymer Chemosensors. J Phys Chem B 2004, 108 (5), 1537-1543.

55. Naguib, Y. M. A.; Steel, C.; Cohen, S. G.; Young, M. A., Triplet-Sensitized Photobleaching of Crystal Violet. J. Photochem. Photobiol. A-Chem. 1996, 96 (1-3), 149-154.

56. Huston, A. L.; Justus, B. L.; Campillo, A. J., Direct Measurement of the Viscosity of Glycerol under Laser Driven Shock Compression - Fluorescence Lifetime Changes in Crystal Violet. Chem. Phys. Lett. 1985, 122 (6), 617-621.

57. Sillen, A.; Engelborghs, Y., The Correct Use of "Average" Fluorescence Parameters. Photochem Photobiol 1998, 67 (5), 475-486.

58. Sadhu, S.; Haldar, K. K.; Patra, A., Size Dependent Resonance Energy Transfer between Semiconductor Quantum Dots and Dye using FRET and Kinetic Model. J Phys Chem C 2010, 114 (9), 3891-3897.

59. Noimark, S.; Salvadori, E.; Gomez-Bombarelli, R.; MacRobert, A. J.; Parkin, I. P.; Kay, C. W. M., Comparative Study of Singlet Oxygen Production by Photosensitiser Dyes Encapsulated in Silicone: Towards Rational Design of Anti-Microbial Surfaces. Phys Chem Chem Phys 2016, 18 (40), 28101-28109.

60. Yaghini, E.; Giuntini, F.; Eggleston, I. M.; Suhling, K.; Seifalian, A. M.; MacRobert, A. J., Fluorescence Lifetime Imaging and FRET-Induced Intracellular Redistribution of Tat-Conjugated Quantum Dot Nanoparticles through Interaction with a Phthalocyanine Photosensitiser. Small 2014, $10(4), 782-792$. 
61. Bovis, M. J.; Noimark, S.; Woodhams, J. H.; Kay, C. W. M.; Weiner, J.; Peveler, W. J.; Correia, A.; Wilson, M.; Allan, E.; Parkin, I. P.; MacRobert, A. J., Photosensitisation Studies of Silicone Polymer Doped with Methylene Blue and Nanogold for Antimicrobial Applications. RSC Adv 2015, 5 (68), 54830-54842.

62. Armstrong, D. A.; Huie, R. E.; Koppenol, W. H.; Lymar, S. V.; Merenyi, G.; Neta, P.; Ruscic, B.; Stanbury, D. M.; Steenken, S.; Wardman, P., Standard Electrode Potentials Involving Radicals in Aqueous Solution: Inorganic Radicals (IUPAC Technical Report). Pure Appl. Chem. 2015, 87 (11-12), 1139-1150.

63. Rao, P. S.; Hayon, E., Reduction of Dyes by Free-Radicals in Solution - Correlation between Reaction-Rate Constants and Redox Potentials. J. Phys. Chem. 1973, 77 (23), 2753-2756.

64. Blackburn, J. L.; Selmarten, D. C.; Ellingson, R. J.; Jones, M.; Micic, O.; Nozik, A. J., Electron and Hole Transfer from Indium Phosphide Quantum Dots. J Phys Chem B 2005, 109 (7), 2625-2631. 65. Reszka, K.; Cruz, F. S.; Docampo, R., Photosensitization by the Trypanocidal Agent Crystal Violet - Type-I versus Type-II Reactions. Chem.-Biol. Interact. 1986, 58 (2), 161-172.

66. Fischer, V.; Harrelson, W. G.; Chignell, C. F.; Mason, R. P., Spectroscopic Studies of Cutaneous Photosensitizing Agents 5. Spin Trapping and Direct Electron-Spin Resonance Investigations of the Photoreduction of Gentian (Crystal) Violet. Photobioch Photobiop 1984, 7 (2), 111-119.

67. Oliveira, C. S.; Turchiello, R.; Kowaltowski, A. J.; Indig, G. L.; Baptista, M. S., Major Determinants of Photoinduced Cell Death: Subcellular Localization versus Photosensitization Efficiency. Free Radic. Biol. Med. 2011, 51 (4), 824-833.

68. Brezova, V.; Pigosova, J.; Havlinova, B.; Dvoranova, D.; Durovic, M., EPR Study of Photochemical Transformations of Triarylmethane Dyes. Dyes Pigment. 2004, 61 (2), 177-198.

69. Bacellar, I. O. L.; Oliyeira, M. C.; Dantas, L. S.; Costa, E. B.; Junqueira, H. C.; Martins, W. K.; Durantini, A. M.; Cosa, G.; Di Mascio, P.; Wainwright, M.; Miotto, R.; Cordeiro, R. M.; Miyamoto, S.; Baptista, M. S., Photosensitized Membrane Permeabilization Requires Contact-Dependent Reactions between Photosensitizer and Lipids. J. Am. Chem. Soc. 2018, 140 (30), 9606-9615. 


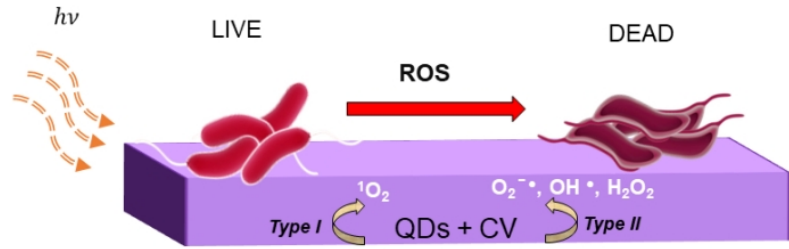

$338 \times 190 \mathrm{~mm}(96 \times 96$ DPI) 